\section{MARCH 2009}

Re-Evolution Summit: Minimal Access Cardiac Surgery

Meeting: March 5-7, 2009, Hotel ZaZa, Houston, Texas. For more information, contact: Veronica Lopez, Office of Continuing Medical Education, 6565 Fannin, MGJ9-004, Houston, TX 77030 (Telephone: 1- 713- 4414971; Fax: 1- 713- 441-0589; E-mail: vlopez2@tmhs.org).

The 17th Annual Meeting of the Asian Society for Cardiovascular and Thoracic Surgery (ASCVTS) and the 2nd AATS/ ASCVTS postgraduate course

Meeting and course: March 5-8, 2009, Taipei International Convention Center, Taipei Taiwan. For more information, contact: Elite Professional Conference Organizer, Ms. Cheryl LIN, 10F-2, No. 51, Sungjiang Road; Taipei 104, Taiwan (Telephone: $+886-$ 2-2504-4338 Ext. 16; Fax: +886-22504-4362; E-mail: ascvts2009@elitep co.com.tw).

$24^{\text {th }}$ International Interventional Cardiology 2009 Symposium

Meeting: March 8-13, 2009, Silvertree Hotel, Snowmass Village, Colorado. Abstract submission deadline: January 23, 2009. For more information, contact: Laurel Steigerwald, 2333 State Street, Suite 203, Carlsbad, California 92008 (Telephone: 1- 760- 7202263; Fax: 1 -760- 720-6263; E-mail: education@promedicacme.com).

\section{$6^{\text {th }}$ Annual Extra-Corporeal}

Membrane Oxygenation Course

Course: March 9-11, 2009, Jeddah, Saudi Arabia. For more information contact: Mr. Faiz Al-Malki (ECMO Coordinator) or Ms. Sonnet Liwanag

\footnotetext{
$\overline{\text { Announcements }}$ of major meetings and other significant activities must be received at least 8 weeks before the desired month of publication. Information will be limited to title of meeting, date, place, and an address to obtain further information. Send announcements to Ryan Walther, Managing Editor, The Journal of Thoracic and Cardiovascular Surgery, 900 Cummings Center, Suite 221-U, Beverly, MA 01915 (E-mail: rwalther@prri.com).
}

(Hospital Assistant), King Faisal Specialist Hospital \& Research Center. MBC-J 16, P.O. Box Jeddah 21449, Saudi Arabia (Telephone: +9662-6677777, ext. 2166 or 5234; Fax: $+9662-$ 663-9581; e-mail: faizwinner@gmail. com; sliwanag@kfshrc.edu.sa).

The Annual Meeting of the General Thoracic Surgical Club

Meeting: March 12-15, 2009, Don Cesar Beach Resort, St. Petersburg, Florida. For more information, contact: Bonnie Lemmerman or Carla MacLean, General Thoracic Surgical Club, Mayo Clinic, 200 First Street SW, Rochester, MN 55905 (Telephone: +1 -507-5384969; Fax: +1 507-284-0058; E-mail: blemmerman@mayo.edu or cmaclean@ mayo.edu)

\section{European School for Cardio- Thoracic Surgery Cardiac \\ Course Level A}

Meeting: March 16-21, 2009, Bergamo, Italy. For more information, contact: EACTS Executive Secretariat, 3 Park St., Windsor, Berkshire S14 1LU, UK (Telephone: +44-1753832-166; Fax: +44-1753-620407; E-mail: info@eacts.co.uk).

\section{Society for Cardiothoracic} Surgery in Great Britain and Ireland 2009 Annual Meeting and Cardiothoracic Forum

Meeting: March 22-24, 2009, Bournemouth International Center, Bournemouth, UK. Further details and abstract submission online at www.scts.org. Contact sctsadmin@scts.org

\section{The Eighth International} Symposium on Sympathetic Surgery (ISSS): Approaching Clinical Care With Clinical Science and VATS 2009: The Seventh International Symposium on Video-Assisted Thoracic Surgery

Combined Meeting: March 25-29, 2009, Sheraton New York Hotel \& Towers, New York, New York. For more information, contact: Melanie
Heath, Associate Program Manager, The CBCE (The Center for Biomedical Continuing Education) (Telephone: 1- 972- 692-2051; Fax: 1- 214- 2600408; E-mail: mheath@thecbce.com).

\section{APRIL 2009}

Functional Ischaemic Mitral Regurgitation (FIMR)

Meeting: April 3, 2009, National Heart and Lung Institute, London United Kingdom. For more information, contact: Karina Dixon, National Heart and Lung Institute, Dovehouse Street, Imperial College, London, SW3 6LY UK (Telephone: +44- 207351- 8172; Fax: +44- 20- 73518246; E-mail: academicevents.nhli@ imperial.ac.uk).

\section{Houston Aortic Symposium:}

Frontiers in Cardiovascular

Diseases - The Second in the Series

Meeting: April 3-5, 2009, The Westin Oaks Hotel, Houston Texas. Abstract submission deadline: February 20, 2009. For more information, contact: Michelle Gherardi, 2333 State Street, Suite 203(Telephone: +1- 760720-2263; Fax: +1- 760- 720-6263; Email:mgherardi@promedicacme.com).

\section{Minimally Invasive Cardiac Surgery}

Meeting: April 17-18, 2009, Sheraton Nicolaus Hotel, Bari, Italy. For more information, contact: Giuseppe Straziota, Department of Cardiovascular and Thoracic Surgery, Anthea Hospital (Telephone: +39- 08- 0- 85644570; Fax: +39- 08- 0- 85644620; E-mail: info@minimally.it).

\section{European School for Cardio- Thoracic Surgery Cardiac Course Level A}

Meeting: April 20-25, 2009, Bergamo, Italy. For more information, contact: EACTS Executive Secretariat, 3 Park St., Windsor, Berkshire S14 1LU, UK (Telephone: +44-1753832-166; Fax: +44-1753-620407; Email: info@eacts.co.uk). 
The International Society for Heart and Lung Transplantation 29th Annual Meeting

Meeting: April 22-25, 2009, Paris France. For more information, contact: International Society for Heart \& Lung Transplantation, 14673 Midway Road, Suite 200, Addison, Texas 75001 (Telephone: 1- 972- 490-9495; Fax: 1- 972 490-9499; E-mail: ishlt@ishlt.org).

The 6th Vienna Interdisciplinary Symposium on Aortic Repair (VISAR)

Symposium: April 22-24, 2009, Congress Venue, Vienna Austria. Abstract submission deadline: February 28, 2009. For more information, contact: Congress Secretariat: E\&E PCO (Telephone: +43- 1- 867- 49- 44; Fax: +43- 1- 867- 49- 44-9; E-mail: office@ee-pco.com).

The 58th International Congress of the European Society of Cardiovascular Surgery (ESCVS)

Congress: April 30 - May 2, 2009 in Warsaw, Poland. Notification of abstract acceptance: January 15, 2009. Low fee pre-registration: March 1, 2009. Scientific Secretariat: Prof. Claudio Muneretto (munerett@med.unibs. it). Organising Secretariat, Meet and Work srl, contact information: Mario Sbalchiero; Telephone +39- 049 -860 1818; FAX +39- 049- 860- 2389; E-mail: meet@meetandwork.com).

\section{MAY 2009}

The 58th International Congress of the European Society for

\section{Cardiovascular Surgery}

Congress: May 1-4, 2009, Warsaw, Poland. For more information, contact: ESCVS General Secretariat, Prof. Claudio Muneretto, ESCVS Secretary General, UDA Cardiochirurgia, Spedali Civili P.le Spedali Civili 1, 25123 Brescia, Italy; (telephone: +39 030 3996401; fax: +39 030 3996096; E-mail: escvs.secretariat@gmail.com; Website: http://www.escvs.org/).
The 8th International

Symposium on Endovascular

Therapeutics

Symposium: May 7-9, 2009, Hotel Hilton, Barcelona, Spain. Abstract submission deadline: February 28, 2009. For more information, contact: Atlanta Unicongress Calvet 55 (Telephone: +34- 902- 109- 853; Fax: +34902- 109- 855; E-mail: site2009@ unicongress.org).

Eighth International Symposium on Redo Cardiac Surgery in Adults

Symposium: May 8-9, 2009 in Boston, Massachusetts. For more information, contact: University of Pittsburgh Medical Center, Division of Cardiac Surgery The Heart, Lung, and Esophageal Surgery Institute, Shadyside Medical Building, Suite 7065200 Centre Avenue Pittsburgh, PA 15232 (Telephone: 1- 412- 6233140; Fax: 1- 412- 623-2822; E-mail: slomkasm@upmc.edu).

\section{The 55th Annual Conference of} the Indian Association of Cardiovascular \& Thoracic Surgeons (CT CON 2009)

Conference: May 14-17, 2009, Srinagar, Kashmir, India. For more information, contact: Conference Secretariat, Prof. AG Ahangar, Head of Department of Cardiovascular \& Thoracic Surgery. Sher-I-Kashmir Institute of Medical Sciences, Soura, Srinagar, India; Tel: +91- 194240- 0348; E-mail: iacts2009@ yahoo.com).

\section{European School for Cardio- Thoracic Surgery Cardiac Course Level B}

Meeting: May 18-23, 2009 Bergamo, Italy. For more information, contact: EACTS Executive Secretariat, 3 Park St., Windsor, Berkshire S14 1LU, UK (Telephone: +44-1753832-166; Fax: +44-1753-620407; E-mail: info@eacts.co.uk).
Postgraduate Graduate Course in General Thoracic Surgery

Course: May 21-22, 2009, Boston, Massachusetts. For more information: Call: (617) 384-8600, 10:00 am-4:00 pm Eastern Time, Monday-Friday; Fax: (617)384-8686; E-mail: hms-cme@ hms.harvard.edu.

The XIV Congress of the Catalan Society for Cardiac Surgery

Congress: May 27-30, 2009, Hotel Avenida Palace, Barcelona Spain. Abstract submission deadline: March 15, 2009. For more information, contact: Nuria Bosch - Oldisseny S.L., Comte (Telephone: +34- 934- 510- 792; Fax: +34- 934- 511- 736; E-mail: nbosch@ oldisseny.com).

\section{5th International Conference on Pediatric Mechanical Circulatory Support Systems \& Pediatric Cardiopulmonary \\ Perfusion}

Conference: May 27-30, 2009, Dallas, Texas. For more information: pedsabstracts@hmc.psu.edu.Website: http://www.hmc.psu.edu/childrens/ pedscpb/

\section{ASAIO - IFAO Annual}

Conference in conjunction with the 5th Annual Pediatric Mechanical Circulatory Support Systems \& Pediatric Cardiopulmonary Perfusion

Conference: May 28-30, 2009, Hilton Anatole Hotel, Dallas, Texas. For more information, contact: ASAIO Inc., 980 North Federal Highway, Suite 212, Boca Raton, Florida 33432-2711 (Telephone: 1- 561- 3918589; Fax: 1 -561- 368-9153; E-mail: info@asaio.com).

\section{3rd Ukrainian-Polish Congress} of Cardiovascular Surgery

Meeting: May 28-29, 2009, Kyiv, Ukraine. For more information, contact: M.M. Amosov National Institute 
of Cardiovascular Surgery of the Academy of Medical Sciences of Ukraine, 6 M.Amosov Street, MSP 03680 (Telephone:: +38 (0)44275-43-22; Fax: +38 (0)44 -27543-22; E-mail: info@amosovinstitute. org.ua).

\section{7th European Conference on}

General Thoracic Surgery

Conference: May 31 - June 3, 2009, Krakow, Poland. For more information, contact: Mondial Congress and Events, Mondial GrmbH \& Co., Operngasse 20b, 10-40 Vienna, Austria; (telephone: +431 58804 0; fax +43 1588040185 ; E-mail: ests2009@mondial-congress. com; Website: http://www.estsmeetings. org/2009/).

\section{JUNE 2009}

Fifth Biennial Meeting of The Society for Heart Valve Disease

Meeting: June 2009, Ritz Carlton Hotel, Berlin Germany. For information, contact: SHVD Executive Secretariat, Heart Science Centre, Harefield Hospital, Harefield Middlesex, UB9 $6 \mathrm{JH}$, UK (telephone: +44 (0)1895828977; fax: +44 (0)1895-828902; E-mail: secretariat@shvd.org; Website: www.shvd.org).

The 2009 International Society for Minimally Invasive Cardiothoracic Surgery (ISMICS) 12th Annual Scientific Meeting

Meeting: June 3-6, 2009, Westin St. Francis in San Francisco, California. For more information, contact: EJ Weldon, 900 Cummings Center, Suite 221-U, Beverly, MA (Telephone: +1 978-927-8330; E-mail: ISMICS@prri.com).

Practices in Cardiac Surgery and Extracorporeal Technologies The New Orleans Conference

Conference: June 10-13, 2009, Westin Canal Place Hotel, New Or- leans, Louisiana. For more information, contact: Joseph Basha, LP, CCP, CEO: Perfusion International, LLC, Program Director: The New Orleans Conference (Telephone: 1318- 623-0890; E-mail: JosephBasha@ PerfusionInternational.com).

European School for Cardio-

Thoracic Surgery Cardiac

Course Level B

Meeting: June 15-20, 2009, Bergamo, Italy. For more information, contact: EACTS Executive Secretariat, 3 Park St., Windsor, Berkshire S14 1LU, UK (Telephone: +44-1753832-166; Fax: +44-1753-620407; E-mail: info@eacts.co.uk).

The 5th World Congress of Paediatric Cardiology and Cardiac Surgery

Congress: June 22-26, 2009, Cairns Convention Centre, Cairns, Queensland Australia. For more information, contact: ICMS Pty Ltd (Telephone: +613- 9682- 0244; Email: pccs2009@ icms.com.au).

The Western Thoracic Surgical Association (WTSA) 35th

\section{Annual Meeting}

Meeting: June 24-29, 2009, Fairmont Banff Springs Hotel, Banff, AB Canada. For more information, contact: April Conti; 900 Cummings Center; Suite 221-U, Beverly, MA 01915 (Telephone: +1 978-927-8330; FAX: +1 978-524-8890; Email: wtsa@prri. com).

\section{JULY 2009}

The 13th World Conference on Lung Cancer (WCLC 2009)

Conference: July 31 - August 4, 2009. San Francisco, Califormia, USA. Abstract Submission Deadline: March 13, 2009. For more information, contact: Khara Robertson, Conference Manager, (telephone: 1604-681-2153, e-mail atwclc2009@ meet-ics.com; Website: http://www. 2009worldlungcancer.org/).

\section{SEPTEMBER 2009}

European School for Cardio-

Thoracic Surgery Cardiac

Course Level C

Meeting: September 21-26, 2009, Bergamo, Italy. For more information, contact: EACTS Executive Secretariat, 3 Park St., Windsor, Berkshire S14 1LU, UK (Telephone: +44-1753-832166; Fax: +44-1753-620407; E-mail: info@eacts.co.uk).

\section{OCTOBER 2009}

Venice 2009 Arrhythmias - 11th International Workshop on

Cardiac Arrhythmias

Workshop: October 4-7, 2009, Fondazione Vini, Venice, Italy. Abstract submission deadline: April 7, 2009. For more information, contact: Rita Reggiani - Project Leader, Rimini, Italy (Telephone: +39- 0541- 305822305811; Fax: +39 -0541-305842; E-mail: infor@venicearrhythmias. org).

The $23^{\text {rd }}$ European Association for Cardio-Thoracic Surgery (EACTS) Annual Meeting

Meeting: October 17-21, 2009, Austria Centre, Vienna, Austria. Abstract submission deadline: April 1, 2009. For more information, contact: EACTS Executive Secretariat, 3 Park St., Windsor, Berkshire Sl4 1LU, UK (Telephone: +44-1753-832-166; Fax:+44-1753-620407; E-mail: info@ eacts.co.uk).

Association of Thoracic and Cardiovascular Surgeons of Asia (ATCSA) 19th Biennial Congress 2009

Congress: October 25-28, 2009, Sheraton Grande Walkerhill Hotel, Seou, Republic of 1 Korea. Abstract submission deadline: June 1, 2009. 
For more information, contact: Ms. Hyo-Shin Nam, InSession International Convention Services, Inc (Phone: +82- 2 -3471 8555; Fax: +82- 2- 521- 8683; E-mail: atcsa2009@insession.co.kr)

\section{Vanderbilt Valve Symposium: 21st Century Techniques for Complex Valve Surgery}

Symposium: October 29-30, 2009, Nashville Marriott Hotel, Nashville, Tennesse. For more information, contact: Joan Lorber, Vanderbilt Heart, Department of Surgery, 1215 21st Avenue South, 5209 MCE, Nashville, TN 37232-8802 (Telephone:1- 615- 3437363; E-mail: joan.lorber@vanderbilt. edu).
NOVEMBER 2009

\section{European School for Cardio- Thoracic Surgery Cardiac Course Level C}

Meeting: November 9-14, 2009, Bergamo, Italy. For more information, contact: EACTS Executive Secretariat, 3 Park St., Windsor, Berkshire S14 1LU, UK (Telephone: +44-1753832-166; Fax: +44-1753-620407; E-mail: info@eacts.co.uk).

The 5th International Meeting of the Onassis Cardiac Surgery Center: Current Trends in Cardiac Surgery and Cardiology Meeting: November 12-14 2009, Athens Hilton Hotel, Athens, Greece. For more information, contact: Liana
Iliopoulou, Triaena Tours \& Congress, 206 Sygrou Avenue, 17672 Athens (Kallithea) Greece (Telephone: +30 -2107499353; Fax: +30- 210- 7705752; E-mail: lianae@triaenatours.gr).

\section{The International Joint Meeting} on Thoracic Surgery

Meeting: November 25-27, 2009, Auditorio Fomento de Trabajo Barcelona, Barcelona Spain. Abstract submission deadline: May 15, 2009. For more information, contact: Oriol Seto, ACTO SERVEIS, C/ Bonaire, 7; 08301 Mataro, Barcelona, Spain (Telephone: +34 -937- 552- 382; Fax: +34 -937- 552- 383; E-mail: thoracic.surgery@actoserveis.com). Additional information: http://www. thoracicsurgery2009.org 